\title{
Expert Discussion: ASCO 2021
}

\author{
Shani Paluch-Shimon ${ }^{a} \quad K^{2}$ aren A. Gelmon ${ }^{b} \quad$ Alexandru Eniuc Joseph Gligorov ${ }^{\mathrm{c}}$ \\ ${ }^{a}$ Breast Oncology Unit, Sharett Institute of Oncology, Hadassah University Hospital and Faculty of Medicine, Hebrew \\ University, Jerusalem, Israel; ${ }^{b}$ Division of Medical Oncology, Department of Medicine, University of British Columbia, \\ Vancouver, BC, Canada; ' Hôpital Riviera-Chablais, Vaud-Valais, Switzerland; d Institut Universitaire de Cancérologie \\ AP-HP, Sorbonne Université, Paris, France
}

\section{Paluch-Shimon: Are PARP Inhibitors Ready for Primetime in EBC (Early Breast Cancer) for Women with a Germline BRCA Mutation? If Yes, in what Clinical Settings, and Do You Have Any Reservations about Its Use in This Setting?}

Gelmon: Yes, PARP inhibitors are ready for primetime in $\mathrm{EBC}$ for persons with germline BRCA mutations.

The results of the Olympia study were presented at the ASCO 2021 meeting and deserved to be chosen for the number one plenary spot. After a median follow-up of 3.5 years for the first 900 women known as the mature cohort, and of 2.5 years for the entire population, there was a clear statistically and clinically significant benefit in disease progression and invasive disease progression in favor of the olaparib arm with a $9 \%$ difference. All groups benefited, including both BRCA1 and BRCA2 as well as hormone positive and triple negative. This was a true demonstration of a target effect in an early setting similar to the effect we saw with the initial studies of trastuzumab in the HER2-positive population.

There will be some challenges integrating this into clinical practice when olaparib is approved. We need to revamp our current guidelines for testing for germline mutations as we currently miss many with hormone-positive cancers and incorporate timely germline testing into our initial new patient consultation for any person who may be a candidate for this treatment. Although the eligibility for the trial required very high-risk cancers, it is more reasonable to consider any stage II or III with a germline mutation as well as somatic mutations and per- sons with germline PALB2. The other questions revolve around other treatments and their effect in these patients including the role of platinums, capecitabine, immune therapy and abemaciclib. Whether olaparib and other agents will be tolerated together or be beneficial as combined therapy is not known.

The results from Jennifer Litton at ASCO 2021 with talazoparib in the neoadjuvant setting with a pathological complete response (pCR) rate of approximately $45 \%$ provides further proof to the role of PARP inhibitors in the early setting although further work needs to be done.

The data are a clear example of a target effect, and although there are no long-term data on efficacy and toxicity, this treatment should be considered for stages II and III breast cancer in persons with germline mutations including cancers that did not achieve a pCR or in the highrisk adjuvant setting.

Eniu: I believe these data from Olympia are practice changing, as they offer a new therapeutic intervention for a meaningful subgroup of patients. However, we are all aware of the complexities of treating breast cancer, as we have to consider interrelations of multiple factors. The BRCA germline mutation is not a hallmark of aggressivity (e.g., POSH study, Copson, Lancet Oncology 2018), therefore it does not highlight a more aggressive subtype of cancer. For the HR+ high-risk population included in the study $(4 \mathrm{~N}+)$, addition of olaparib on top of standard adjuvant hormone therapy and bisphosphonates should be discussed, although this subgroup represented only less than $20 \%$ of the trial population, and, albeit exploratory, the subgroup analysis raises questions about the 
magnitude of benefit in this subpopulation. Issues of compliance to oral treatment will be important. For the triple negative breast cancer (TNBC) subgroup, in the non-pCR postneoadjuvant subgroup, standard therapy with adjuvant capecitabine (Create-X trial) improves overall survival, therefore this is the standard of care and currently it is unknown how olaparib compares with capecitabine in the adjuvant setting (only data from a metastatic setting). Therefore, the TNBC subgroup for which olaparib provides an indisputable straightforward indication are TNBC that received surgery upfront, carrying a gBRCA mutation, $\geq \mathrm{pT} 2, \geq \mathrm{pN} 1$ as included in the study design.

Gligorov: Yes, in an adjuvant setting for all patients with a "high risk" of relapse that means:

- Initially pN+

- No pCR if neaodjuvant treatment

Of particular concern is the fatigue and the anorexia in some patients that might be a limiting adverse event for 1 -year treatment.

\section{Paluch-Shimon: What Factors Guide Your Decision on Extended Adjuvant Hormonal Therapy in EBC, and Will Results Presented at ASCO 2021 Change how You Decide?}

Gelmon: Since extended adjuvant hormonal therapy was first shown to impact outcomes, studies have tried to find markers to determine who benefits as obviously long-term therapy has toxicity and therefore it is important to determine whether there are persons who could be spared 10 years of treatment.

Two translational studies using samples from B42 tried to determine whether there were markers. Dr. Mamounas presented data on 2,179 tumors in the translational cohort assessed by BCI which is an 11-gene molecular signature that had been shown in previous analyses to be predictive of late relapse. BCI was reviewed and did not show a predictive value for BCI in terms of recurrence-free interval which was the time from randomization to breast cancer recurrence and compared the low and high BCI scores.

The translational cohort from B42 was also reviewed in terms of the predictive value for Mammoprint. This analysis showed that persons with low Mammoprint levels did have a benefit for extended adjuvant therapy with a $7.8 \%$ difference for disease-free survival (time from randomization to breast cancer recurrence, other primary malignancy or death) and a 7.0\% difference for breast cancer-free interval (time from randomization to either distant recurrence or contralateral breast cancer). However, again as a large number of persons in this trial developed contralateral breast cancer as their event, this may affect the results of a molecular assessment of the first primary as a predictor for relapse.

In summary, it appears that clinical features continue to be the best indicator for extended adjuvant hormonal therapy. High-risk features such as nodal involvement or stage remain risks for late relapse including in low molecular cancers. The decision to continue therapy is therefore made on these factors coupled with the person's tolerance to therapy and the treatment's impact on quality of life. BCI, Mammoprint and other molecular tests need further assessment prior to being used in standard clinical practice.

Eniu: Published evidence, including data from NSABP B42 and MA17r, suggest that the benefit of extended aromatase inhibitor therapy up to 10 years is limited (3-4\%). Approximately half of the benefit seen in these trials comes from preventing events not linked to the initial cancer treated (contralateral cancers, 2 nd cancers), therefore genomic testing of the previous cancer might not be informative for this type of events. Additionally, neither Mammoprint nor BCI data presented at ASCO provide compelling evidence of their utility in predicting a benefit of extended aromatase inhibitor therapy. In my opinion, we are left still with the need to thoroughly weight and discuss potential benefits versus toxicity with our patients based on classical parameters such as age, tumor burden, previous tolerance with aromatase inhibitors and bone density data.

Gligorov: The main two factors are risk of relapse (essentially node positivity) and endocrine sensitivity (predictive factor for prolonged treatment). ASCO 2021 results will not change my way of decision or treatment actually.

\section{Paluch-Shimon: What Are Your Take-Home Messages from the Results of the WGS ADAPT Studies for Escalating and De-Escalating Treatment in (i) HER2+ EBC and (ii) Luminal EBC?}

Gelmon: The ADAPT HER2 study reported on the longer-term outcomes of persons treated with a 12-week regimen of pertuzumab/trastuzumab and the same protocol with the addition of weekly paclitaxel. This study is very interesting in terms of de-escalating therapy. The results are another example of the prognostic role of pCR. The pCR rate was significantly better in the group who received paclitaxel, and of interest $79 \%$ of those on the chemo arm and $29 \%$ on the no-chemo arm received no further therapy after their surgery.

This study suggested that further therapy after a pCR had been achieved was not needed as these persons had excellent outcomes. The study was not large so there may be some uncertainty about the actual pCR rate with a che- 
mo-free regimen. As well, the results are in ER-negative HER2-positive cancers which are more responsive to anti-HER2 therapy and chemotherapy than ER-positive HER2-positive tumors. However, this study provides interesting data on de-escalating therapy and on using the response of the individual tumor as the guide to the subsequent treatment. The investigators also defined that the HER2-enriched subtype is the sensitive subtype as compared to their nonenriched tumors.

Dr. Gluz presented a trial for patients with hormonepositive cancer who would have been candidates for chemotherapy according to standard clinical parameters, the ADAPT HR+ HER2 - trial. This trial assessed a dynamic Ki67 as well as the recurrence score.

Those patients without a response to endocrine therapy who went on to receive chemotherapy and had a recurrence score less than 25 had similar good outcomes when compared to the endocrine responsive patients who were spared chemotherapy. However, those younger than 50 years had more benefit from the addition of chemotherapy than those older than 50 . The recurrence score was very predictive of outcome for the $\mathrm{N} 2$ and $\mathrm{N} 3$ patients regardless of endocrine responsiveness. Likewise for those with recurrence scores greater than 25 , nodal status and tumor size were predictive of outcome.

\section{Take-Home Messages}

In responsive HER2-positive tumors, pCR may predict a good outcome and provide a basis for de-escalation of treatment, and this has been shown with the longer follow-up of the ADAPT study.

In hormone-positive, HER2-negative patients, dynamic Ki67 may help provide evidence for a group of patients who have a good outcome with endocrine therapy alone despite clinical features suggesting high risk. However, tumor size and nodal status still do impact outcomes for those with higher-risk disease. Age less than 50 continues to show different outcomes compared with age $>50$ despite similar recurrence scores. New options for therapy are needed for persons with endocrine positive tumors which have molecular and clinical high-risk features.

Eniu: The data from the WGS ADAPT trial are intellectually fascinating as it investigates the concept of integrating early in the treatment decision parameters (clinical risk, genomic risk and proliferation) that were classically used after surgery to decide upon adjuvant systemic therapy. For luminal cancers, it also provides further data on the role of "endocrine response" (Ki67 data after 2 weeks of endocrine therapy) on prognosis, allowing perhaps in the future de-escalation strategies, pending rigorous validation of on-therapy biopsy parameters. The data on the utility of the recurrence score in patients with more than 4 positive nodes to identify low RS patients that have an excellent prognosis is interesting but needs larger val- idation before use in the clinical setting. The WGS ADAPT HER2+ study focused on de-escalating the chemotherapy component in the RH-HER2-positive subgroup, which likely includes predominantly HER2-enriched tumors and across trials has shown superior response and outcomes to anti-HER2 therapy. Although these data are not practice-changing, the results reinforce the concept that less chemotherapy might be sufficient in HER2-driven tumors.

Gligorov: Overall, it is possible to predict very early at initiation of the treatments the probability of pCR.

Concerning HER2+, the optimal regimen without anthracyclines includes double blockade with taxanes. It is not yet ready for de-escalation.

As for luminal EBC, using OncotypeDx and early prediction of pCR might be interesting to improve treatment strategies by using endocrine treatment alone or switch to chemotherapy accordingly to the initial risk ratio and impact of endocrine treatment on an early decrease in Ki67.

\section{Paluch-Shimon: In Light of Results Presented at ASCO 2021, What Is Your Treatment Algorithm for Managing TNBC in Early Stage 2-3 Breast Cancer?}

Gelmon: After the CREATEx trial had been published there were questions about whether capecitabine was the best drug to use for residual disease, especially as there is ongoing discussion about the role of carboplatin or cisplatin in TNBC in the neoadjuvant setting. The ECOG ACRIN trial EA1131 randomized persons after neoadjuvant treatment who did not achieve a pCR to test whether one of these agents was superior in the adjuvant setting. It was stopped early for futility with only 308 patients enrolled when it became clear that it would not demonstrate noninferiority or superiority. This study did suggest that capecitabine should be recommended in ER-negative tumors that do not achieve a pCR and are not germline carriers or candidates for PARP inhibitors.

There were further data on PDL1 inhibitors in the TNBC with longer follow-up from the GeparNuevo trial which enrolled 174 patients and investigated the addition of durvalumab, an anti-PDL1 checkpoint inhibitor, to standard neoadjuvant chemotherapy in patients with early TNBC. Durvalumab modestly increased the pCR rate to $53.4 \%$ versus placebo $44.2 \%$. However, this study reported that durvalumab added to neoadjuvant chemotherapy in TNBC significantly improved long-term outcome despite a small pCR increase and no continuation after surgery. The authors appropriately question whether adjuvant therapy with immune checkpoint inhibitors is needed at all. 
As for new algorithms for TNBC, in the TNBC space we are now looking at adding in immune checkpoint inhibitors to improve pCR for those patients who may respond in the neoadjuvant setting but possibly not continuing it into the adjuvant setting. Data suggest that we should continue to recommend capecitabine after neoadjuvant therapy as it remains the most tested option to provide an overall survival advantage. For patients with germline mutations we should include adding PARP inhibitors in the adjuvant setting.

Eniu: Considering the evidence available so far, in a nutshell my position is that we are not yet ready to incorporate immune therapy as part of the management of early TNBC. The format of this article does not allow for a full discussion of data, but as of today there are still more questions than answers regarding the best way to integrate checkpoint inhibitors in standard clinical use in the neoadjuvant setting, their benefit as adjuvant treatment as well as long-term efficacy and safety data. The role of platinum as postneoadjuvant therapy in TNBC could not be established as per the EA1131 trial, therefore the current standard remains capecitabine, with the additional role of olaparib as highlighted above.

Gligorov: I recommend neoadjuvant chemotherapy dose dense $\mathrm{AC} \times 4$ followed by weekly paclitaxel, germline BRCA mutation screening during this period, then after surgery:

\begin{tabular}{llll}
\hline & & $\begin{array}{l}\text { Initial high risk } \\
(\text { essentially } \mathrm{pN}+)\end{array}$ & $\begin{array}{l}\text { Initial lowest risk } \\
(\mathrm{pN0})\end{array}$ \\
\hline $\mathrm{pCR}$ & BRCA mut & Adjuvant olaparib & $\begin{array}{l}\text { No adjuvant } \\
\text { treatment } \\
\text { No adjuvant } \\
\text { treatment }\end{array}$ \\
\hline No pCR & $\begin{array}{lll}\text { BRCA mut } m \text { to adjuvant } \\
\text { BRCA } w t\end{array}$ & $\begin{array}{l}\text { Adjuvant olaparib } \\
\text { Adjuvant } \\
\text { capecitabine }\end{array}$ & $\begin{array}{l}\text { Adjuvant olaparib } \\
\text { Adjuvant } \\
\text { capecitabine }\end{array}$ \\
\hline
\end{tabular}

\section{Conflict of Interest Statement}

Shani Paluch-Shimon: advisory boards, consultancy, speaker's bureau of Pfizer, Novartis, Astra Zeneca, Lilly, Exact Sciences, Nanostring, Roche. Karen A. Gelmon: advisory boards of Pfizer, Novartis, Astra Zeneca, Lilly, Merck, Nanostring, Genomic Health, BMS, Roche, Mylan, Ayala; research funding from BMS, Pfizer, Novartis, Roche, AstraZeneca; expert testimony for Genentech. Alexandru Eniu: received research support from AstraZeneca, Roche, Pfizer, Celltrion, Novartis; none in relation to this presentation. Joseph Gligorov: Astra Zeneca, Daiichi, Esai, Exact Science, Lilly, Merck, Novartis, Pfizer, Roche, Seattle Genetics.

\section{Chair}

Dr. Shani Paluch-Shimon

Breast Oncology Unit

Sharett Institute of Oncology

Hadassah University Hospital

Jerusalem (Israel)

shanipal@hadassah.org.il

\section{Participants}

Prof. Karen A. Gelmon

BC Cancer

600 West 10th Avenue

Vancouver, BC, V5Z 4E6 (Canada)

kgelmon@bccancer.bc.ca

Dr. Alexandru Eniu

Breast Medical Oncologist

Coordinator, Oncology Pole

Hôpital Riviera-Chablais, Vaud-Valais

Route du Vieux-Séquoia 20

CH-1847 Rennaz (Switzerland)

aeniu@eso.net

Prof. Joseph Gligorov

Hôpital Tenon, Oncologie Médicale

4 , rue de la Chine

FR-75020 Paris (France)

joseph.gligorov@aphp.fr 